\title{
Bosch loT Suite: Exploiting the Potential of Smart Connected Products
}

\author{
Sven Jung, Stefan Ferber, Irene Cramer, \\ Wolfgang Bronner, and Felix Wortmann
}

\section{Towards Smart Connected Products}

The Internet of Things (IoT), i.e. the convergence of the physical and digital world, promises enormous business potential and will have a lasting impact on every industry (Iansiti and Lakhani 2014; Porter and Heppelmann 2014, 2015). Specifically, smart, connected products will change the way how manufacturing companies create and capture value in both B2B and B2C (Porter and Heppelmann 2014, 2015). Well-known examples of manufacturing

\author{
S. Jung (四) \\ University of St. Gallen, St. Gallen, Switzerland \\ e-mail: sven.jung@unisg.ch \\ S. Ferber • I. Cramer \\ Robert Bosch GmbH, Stuttgart, Germany \\ e-mail: stefan.ferber@bosch-si.com; rene.cramer@softwareag.com \\ W. Bronner
}

Bosch IoT Lab at University of St. Gallen \& ETH Zurich, St. Gallen, Switzerland e-mail: wolfgang.bronner@bosch.com

F. Wortmann

University of St. Gallen, Institute of Technology Management,

St. Gallen, Switzerland

e-mail: felix.wortmann@unisg.ch 
companies that are already successfully using the IoT today are Tesla (connected cars) in automotive, Philips (connected lighting) and Sonos (connected speakers) in smart home as well as John Deere (connected tractors) in agriculture and Trumpf (connected machines) in the domain of Industry 4.0.

At the same time, many manufacturing companies find it challenging to develop successful IoT offerings. Oftentimes, their expectations in terms of revenue and profit remain unmet (Wortmann et al. 2019). A recent study from 2020 showed that $58 \%$ of IoT projects are either mostly or completely unsuccessful (Beecham Research 2020). From a provider perspective, the reasons for failure vary from internal challenges (e.g. lacking capabilities) to external ones (e.g. immature technology). However, one very common challenge is the lack of business focus. Companies concentrate on technology and lose sight of the focal business problem they are trying to solve for their customers. In fact, many companies still search for compelling and financially viable use cases that go beyond 'chasing the cool factor' (Kranz 2017). In light of the depicted challenges, this chapter is focusing on IoT and manufacturing companies and addresses three fundamental questions: What are the recurring and proven IoT use cases across different manufacturing industries? How can they be leveraged by manufacturing companies? And what role do IoT platforms play in enabling these use cases?

\section{Internet of Things for Manufacturing Companies}

In the context of manufacturing, the term industrial IoT (IIoT) is omnipresent. However, the lines between IIoT and IoT are very blurry and the concepts are often used interchangeably. IIoT refers to the specific application of IoT in an industrial and B2B setting (Boyes et al. 2018). One prominent example is the connected factory (Industry 4.0) where machine-to-machine communication and automation play an important role. Despite different perspectives on IoT, utilizing IoT relies on common fundamental principles across manufacturing companies independent from the domain. Every IoT solution is based on a smart, connected product such as an industrial machine or smart speaker. The objective is to complement a physical product or device (e.g. tractor) with software and sensors (e.g. to track the GPS and engine data of a tractor) to make it 'smart'. Connecting the smart device with the Internet enables different levels of capability: Smart, connected products can be remotely (a) monitored, 
(b) controlled or (c) optimized. Depending on the domain, they can also operate (d) autonomously (Porter and Heppelmann 2014).

In the long term, the Io $T$ can enable manufacturing companies to offer their customers a hybrid value proposition that is both physical and digital. This usually includes the analysis of data (gathered through the connected products) to offer value-adding digital services (Fleisch et al. 2014). However, every IoT solution must ultimately solve an unmet need for either the manufacturing company itself or the end customer. Depending on the data source, the literature distinguishes four ways in which IoT data can be used by manufacturing companies (Bilgeri et al. 2019) (Fig. 1).

The first two opportunities relate to smart, connected products, which are not owned by the customer but are in the direct sphere of influence of the respective company. Agricultural service providers that provide harvesting services to farmers, for example, use IoT data from their agricultural machinery to improve the efficiency of their internal processes (domain 1). Telemetry data enables them to monitor the position and operating status of their agricultural machinery and optimize their fleet management. Beyond internal process innovation, the telemetry data can also be used for new products and services. The use of highly specialized, high-performance agricultural machinery has become so expensive that customers require precise minute-by-minute billing. Telemetry data enables such billing, which is perceived as a real value add and thus a differentiating service (domain 2). Connecting agricultural

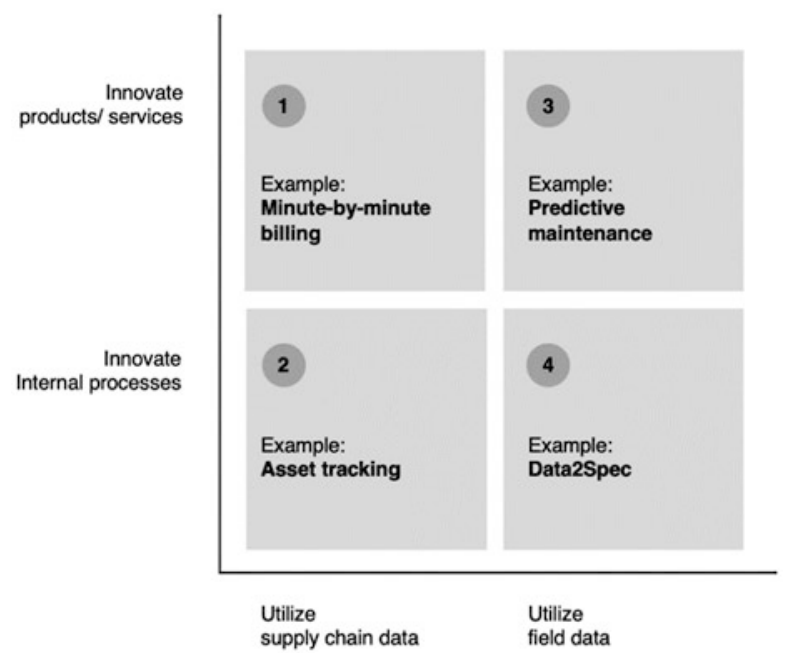

Fig. 1 Four domains to utilize loT data, Bilgeri et al. (2019) 
machinery thereby leads to internal process optimization as well as service innovation for the customer.

The third and fourth opportunity is based on data that is generated by products in the field, i.e. while in use by the customer. Heidelberger can serve as an example to illustrate these opportunities. With its connected solutions, the manufacturer of industrial printing systems is in a position to offer innovative services such as predictive maintenance (domain 3). In the event of a malfunction, the system (located at a customer site) automatically sends an electronic error message to Heidelberger, enabling service technicians to carry out repair work remotely or to bring the right spare parts directly to the site if necessary. The monetization of this new data-driven service offering, however, covers only part of the IoT potential of connected printing equipment. At the same time, the IoT data is also used to optimize the development processes of Heidelberger (domain 4). In the data-to-specification (Data2Spec) use case, historic machine data that captures real-world machine usage serves as the basis to prevent costly under- or over-specification of new product generations. Hence, the connectivity of printing machines also leads to service innovation for the customer as well as internal process optimization at Heidelberger.

Given the technological possibilities and the increasing number of success stories, there is little doubt that the IoT will transform many industries in the long term. It will alter the way companies design and develop their products and services. At the same time, a new technology stack is emerging, and manufacturing companies need new skills to successfully deliver IoT solutions (Porter and Heppelmann 2015).

\section{Bosch loT Suite as an loT Platform}

IoT platforms have become an integral part of IoT projects. Their goal is to provide a manufacturing company with the necessary technology to quickly build and scale an IoT solution. However, the nature of IoT platforms is quite diverse. The value generation of IoT platforms for manufacturing companies can range from very generic (e.g. generic cloud storage) to very specific (e.g. remote update capabilities on the application level) services. Three fundamental IoT platform layers can be distinguished (Fig. 2): infrastructure-as-aservice (IaaS), platform-as-a-service ( $\mathrm{PaaS})$ and software-as-a-service ( $\mathrm{SaaS})$.

Smart, connected products form the basis for every IoT solution (level 0 ). The first IoT platform level corresponds to generic cloud services such as cloud storage and computing (storage and computing can also take place on the device itself). This level is often referred to as the infrastructure level (level 


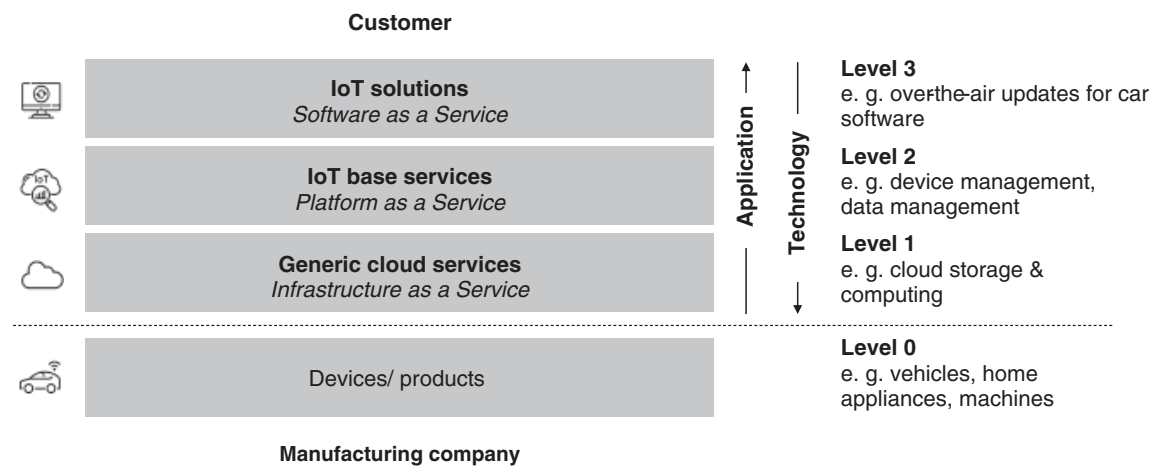

Fig. 2 New loT technology stack and corresponding loT platform levels, author's own illustration

1). The next level corresponds to IoT base services (level 2). Offered as PaaS, these services range from the management of devices to the management of data. Applications solving a concrete need for the end-user are built on top of these two levels. The solution level (level 3) is typically very specific to one industry or customer. This could be, for example, a SaaS solution specifically developed for a manufacturer of cars (OEM) to remotely update its large fleet of cars across the globe securely and reliably.

The importance of IoT platforms for developing ready-to-use IoT solutions has led to a strategic buy-or-build question for manufacturing companies. One can either build their own IoT platform or source a third-party platform. Indeed, many large manufacturing companies have developed their own IoT platform to be more independent of third-party providers. In addition, they are also trying to establish themselves as a provider of an IoT platform. For instance, Bosch launched its own IoT platform with the Bosch IoT Suite in 2013 as one of the first manufacturing companies, alongside General Electric with Predix and Siemens with MindSphere. However, over the last years, many IoT platforms have emerged (150+ and counting) creating a very diverse set of IoT platforms (Graf et al. 2018; Hodapp et al. 2019). This diverse competitive landscape makes it increasingly difficult for a manufacturing company to navigate through. Looking at today's market, four main types of IoT platform providers can be distinguished:

1. Companies with a background in manufacturing (e.g. Bosch, Hitachi).

2. Companies with a background in Product-Lifecycle Management software (e.g. PTC, Siemens).

3. Companies with a pure software background (e.g. Software $A G$ ).

4. Hyper-scalers with a background in generic cloud services (e.g. AWS, Microsoft). 


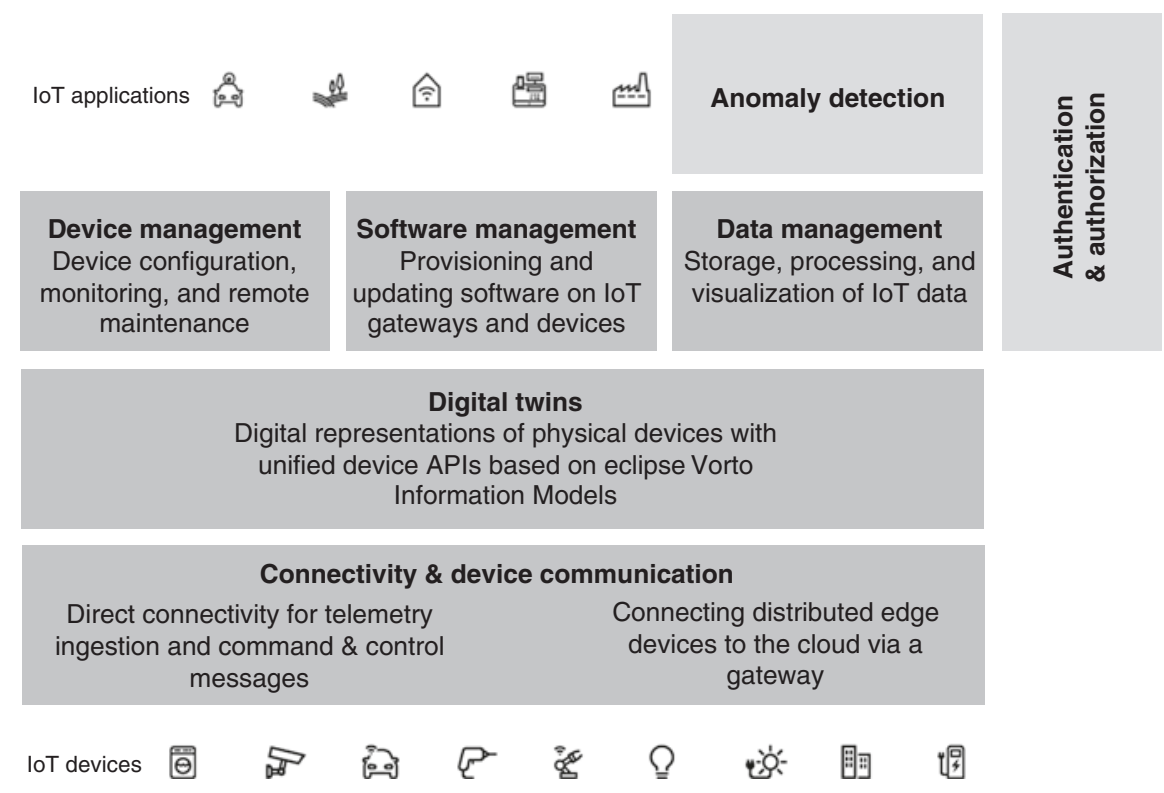

Fig. 3 Bosch loT Suite services on the PaaS level, Bosch.IO (2020b)

As part of the first group, the Bosch IoT Suite, operated by Bosch.IO, serves as an IoT platform for Bosch internally and external customers. Bosch.IO is an independent subsidiary that supports Bosch business units and external customers in their digitalization efforts by providing IoT (software) solutions, such as the Bosch IoT Suite. Specifically, the Bosch IoT Suite focuses on scalable IoT services offered as PaaS, ranging from software for device connectivity and communication to device and data management (Fig. 3). As of today, already more than ten million devices are connected on the basis of the Bosch IoT Suite (Bosch.IO 2020a). On the infrastructure level, Bosch offers its own cloud service but also enables hybrid cloud set-ups. Hybrid cloud set-ups combine third-party cloud solutions (e.g. AWS or Microsoft Azure) with IoT services provided by the Bosch IoT Suite. Also, industry-and customer-specific use cases are developed. To distinguish itself from other IoT platform providers, the Bosch IoT Suite builds on three strategic pillars:

- Developing open-source software: Since early on, Bosch has been a member of the Eclipse IoT working group and has set up several open-source projects. Today, the majority of the Bosch IoT Suite is based on open-source software. The decision to provide an open-source IoT platform has several advantages. A strong developer community can lead to better quality in the 
software benefiting both Bosch and its customers. It also provides speed and cost advantages for Bosch as an IoT platform provider. At the same time, Bosch customers remain independent (from one provider).

- Leveraging industry knowledge: With a long history as a manufacturing company and supplier in various industries, Bosch.IO can build on the strong domain know-how of the entire Bosch Group. This know-how (e.g. in automotive and household appliances) benefits also customers of the Bosch IoT Suite as Bosch.IO can develop applications tailored to the needs of a well-known domain.

- Innovating on proven IoT solutions: The Bosch IoT Suite is used internally by the business units to connect and innovate their products. Today, $92 \%$ of all Bosch electronic product classes can be connected to the Internet (Denner, 2020). Using the IoT platform internally to become a digital manufacturer has provided Bosch.IO with a comprehensive set of proven IoT solutions. These proven solutions are also offered to its customers enabling them to 'manufacture like a Bosch'.

\section{$4 \quad$ Six Core loT Use Cases for Manufacturing Companies}

In the last years, Io $\mathrm{T}$ base services such as IoT device management have become more and more a commodity. Hence, attractive use cases on the application level-that go beyond the 'cool' factor-became the new source of competitive advantage. In light of this development, we looked at success stories at Bosch as well as its customers to identify the most attractive and replicable IoT use cases across industries. In recognition of Bosch's rich history in manufacturing, we focused our analysis on those IoT use cases that provide an added value for manufacturers of discrete products. Through our analysis, we have derived six cross-domain (horizontal) IoT use cases which aim to solve the following needs for a manufacturing company across domains (Fig. 4):

(a) Manufacturers want to provide better products and services to their customers.

(b) Manufacturers want to increase their customers' productivity and experience.

(c) Manufacturers want to continuously improve and innovate their portfolio. 


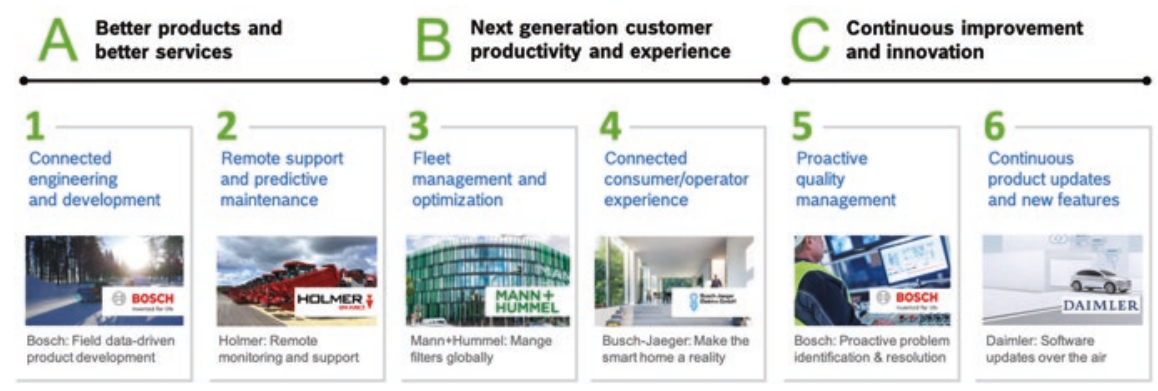

Fig. 4 Framework with six horizontal loT use cases, author's own illustration

The first two horizontal IoT use cases help manufacturing companies to improve their products and services. This is primarily enabled by remote access to their smart, connected products in the field.

\subsection{Connected Engineering and Development}

\section{Description}

Operational data of devices in the field such as information on device status, usage or failures is used to define future product specifications. Rather than relying on assumptions of how a product might be used in reality, actual field data provides companies with a more accurate picture of the actual use of a particular product in the field. This transparency helps companies to design and develop better products in the long run. For instance, by connecting car brakes to the Internet, Bosch was able to measure the actual hydraulic load in a particular car model. This unique insight enabled Bosch to use an existing braking system instead of modifying the system as recommended by existing validation models, which were only based on assumed loads. The additional insights prevented Bosch from over-engineering the new generation of brakes. Furthermore, loT field data and remote access can be used to accelerate and improve product development and validation. Product innovations in the pipeline can be tested, validated and adapted based on models fed with field data-instead of models only based on assumptions.

\section{Business Value}

The use case 'connected engineering and development' helps manufacturing companies to reduce cost arising from over-specification of products. As companies typically design products based on a variety of assumptions, products often end up being over-specified, i.e. with features that are not necessarily needed or desired by the end-user. At the same time, this use case helps companies to avoid unsatisfied customers due to under-specification of the respective products. Last but not least, it can shorten time-to-market and development cycles, especially in the validation phase of a new product in the pipeline. 


\subsection{Remote Support and Predictive Maintenance}

\section{Description}

While the first use case aims to use loT technology at an early stage of the value chain (i.e. in product design and development), the second loT use case aims to improve (after-sales) service. This is based on the condition that not only the customer but also the manufacturer can monitor and access products in the field remotely. This offers the manufacturer many opportunities to make its service efforts more effective and efficient. Products can be monitored and inspected remotely by the manufacturer without the need for an on-site presence. With this, instant support for the customer can be provided if products or machines fail. Also, service technicians can call up data on the condition of the equipment. In this way, the service technicians are kept up to date on which components need to be repaired or which spare parts are required. Furthermore, manufacturing companies can leverage data from the connected products enabling predictive or condition-based maintenance. Instead of repairing a machine, the goal is to service a machine before a potential failure occurs. By analysing historic product failures, patterns indicating a potential failure can be identified. For instance, if analyses show that the temperature just before a machine failure has always exceeded a certain threshold, this pattern can be used to foresee (or prevent) a future failure.

\section{Business Value}

The use case 'remote support and predictive maintenance' helps manufacturing companies to avoid long-repair cycles and machine downtimes at the customer site. This is particularly beneficial for the customer as downtimes typically have a direct, negative financial impact on the customer's business. At the same time, this use case eliminates the cost of manual on-site inspections for the manufacturer, as the majority of the service work can be carried out remotely. In the long run, it ensures cost-effective and preventive maintenance, from which both the manufacturer and the customer can benefit greatly. The next category of horizontal loT use cases aims to benefit the direct user of the machine or product. The goal of both use cases is to create a better experience for the end-user and increase the customer's productivity.

\subsection{Fleet Management and Optimization}

\section{Description}

The first use case of this category provides value to the particular user group that is not using a single device but managing an entire fleet of devices. This could be, for example, a dispatcher in the context of logistics, who manages a fleet of 
(continued)

trucks, or a manager in the context of a factory, who manages a fleet of machines. By connecting an entire fleet of devices, fleet managers can remotely check the status of the devices (e.g. location, health, usage) or benchmark the performance of individual devices and machines. Typically, a dashboard or desktop application is provided which enables intuitive use by the customer. The objective is to enable simple monitoring of devices and machine states. This, in turn, enables easy benchmarking across the fleet to identify best practices and well-/bad-performing machines. Typically, integrated into the existing IT systems, fleet managers can seamlessly order spare parts and consumables as part of this use case.

\section{Business Value}

With the use case 'fleet management and optimization', manufacturing companies can increase their customers' productivity. It primarily increases transparency for the customer's fleet manager. In particular, it allows fleet managers to optimize the performance of individual devices or increase asset utilization across the entire fleet as well as decrease administrative costs.

\subsection{Connected Consumer/Operator Experience}

\section{Description}

The second use case of this category provides value to the individual user of a product or machine. In a B2C context, this could be a homeowner, but in a B2B context, this can also be a machine operator on the shop floor. The objective for a manufacturer is to complement the smart, connected products with a digital experience for the end-user. In comparison to use case 3 , this use case is about the individual device and not a fleet of devices. Moreover, the focus is not on a central fleet manager but on a local operator. This is why this use case typically includes a user interface with functionality to monitor and control single devices (e.g. kitchen appliances, lighting or sound in the smart home). Thereby, consumers (B2C), as well as operators (B2B), are being empowered to optimize their device usage and they profit from increased convenience. This can be supported by offering data-driven advice or a remote assistant (see loT use case 2). For instance, Daimler offers a smartphone application that is connected with the car. With the app, users can remotely review the status of the car (e.g. location, driven mileage, petrol left, warranty) and perform both simple actions like closing or opening the car and more complex actions such as remote car parking via the smartphone (Mercedes 2020). 


\section{Business Value}

With the use case 'connected customer/operator experience', manufacturing companies can open up new revenue streams or secure the existing ones. Given the increasing demand for digital product experiences, this use case ensures that consumer expectations are met and enables manufacturing companies to stay competitive. Also, it helps consumers and operators to become more efficient and productive in their product usage.

The third category of horizontal loT use cases aims at improving and innovating a company's product portfolio-both the products that are currently in the field and the new product generation that is still under development.

\subsection{Proactive Quality Management}

\section{Description}

Without the use of IoT, manufacturing companies have to collect information on individual product failures or warranty issues from their distributors or repair shops. This is a very costly approach and relevant information can get easily lost in the long process of consolidation. Due to device connectivity, manufacturing companies can now collect this same data remotely and investigate product problems much faster and more reliably. This allows for a much better problem and quality management (Quality 4.0). The objective is to identify potential, systematic problems across the entire value chain, from sourcing, design, to manufacturing and sales. By automatically collecting failure information from connected devices in the field, the manufacturing company can respond to systematic failures and possibly find the root cause in the value chain much earlier. This, in turn, allows for fast adjustments of the product specification and early customer communication (e.g. to prevent dissatisfied customers and public recalls). In the long run, it can also help set up servicelevel agreements between customer and manufacturer without the need for a middleman.

\section{Business Value}

The use case 'proactive quality management' can significantly increase customer satisfaction. By detecting systematic problems earlier than before, potential recalls including penalties as well as warranty costs can be avoided. In the long run, this helps to increase the quality of the products across the entire value chain. 


\subsection{Continuous Product Updates and New Features}

\section{Description}

Originally, to be updated, products had to be brought into a dealership which was a cumbersome and lengthy process for the customer. Due to device connectivity, manufacturers can now remotely update embedded firm and software of devices in the field (and even while being in use by the customer). Besides updating the existing firm and software, this also allows manufacturing companies to remotely upload new applications or functionalities to a device. This 'smartphone-like' approach becomes more and more important for traditional product manufacturers. For example, the Bosch IoT Suite enables Daimler to remotely update the firmware of roughly four million Mercedes cars worldwide (Bosch.IO n.d.). Via the cellular network, the infotainment system (e.g. navigation maps) or even security and safety-relevant components of the cars can be updated ensuring consistent customer experience. In turn, this means that car owners no longer have to visit their car dealer to receive an update for their car.

\section{Business Value}

With the use case 'continuous product updates and new features', manufacturing companies can sustain (and even increase) the product lifetime and value for the customer. It can assure device security by updating to the newest software and even increase revenue by adding new functionalities after the product has been sold to the customer. In addition, updating products and devices that are already deployed in the field is often considered the foundation for many of the other mentioned loT use cases. As part of remote services, it can support managing product failure as the firmware can be updated to fix a bug. Overall, this horizontal loT use case can lead to new revenue opportunities for the manufacturer while increasing customer satisfaction at the same time.

\section{$5 \quad$ Managerial Implications}

The six IoT use cases have showcased the great potential for manufacturing companies across domains. By leveraging IoT, manufacturing companies can make their products and services better, increase customers' productivity and innovate their product portfolio in the long run. Many recent studies, however, also suggest that most IoT projects and initiative still fail. We, therefore, want to highlight seven takeaways for manufacturing companies that want to embark on an IoT journey: 
1. Systematically assess your IoT opportunities: In a first step, screen your own as well as adjacent industries for successful IoT solutions and identify the most important trends. Companies often focus too much on their own industry while overlooking competitors from adjacent markets building cross-industry IoT solutions. Understanding the potential of IoT for your business also requires you to look closely at your own hardware portfolio. Based on this internal and external analysis, evaluate systematically the chances and risks for your Io $T$ endeavour.

2. Define your own role and think about partnerships: Doing IoT alone becomes increasingly difficult due to the complex technology stack needed. In fact, most IoT solutions require close collaboration among several companies. It is therefore important for you to define early on what role you want to play in this ecosystem. Based on this, analyse what you can develop in-house, where you need a supplier and where you want to strategically partner up with other leading companies (e.g. IoT cloud or software providers).

3. Stay true to your core: Most companies follow mainstream and focus on 'catchy' IoT use cases that are heavily discussed in news or marketing. Because they are hyped, they initially seem promising, but often do not end up as successful as expected. Manufacturing companies should always start by selecting those IoT use cases that are close to their core business. In addition, they should focus on proven Io $T$ use cases that have the potential to generate short-term profits.

4. Customer value first, technology second: Many IoT products result in low adoption when the manufacturing companies focus on technology improvements instead of building compelling use cases for their customers. Therefore, start with addressing a concrete customer or business problem. Only then think about how the use of IoT technology can help to solve this problem. Our set of six horizontal IoT use cases can help to embrace such use-case focus.

5. Have a holistic IoT strategy in mind but prioritize: Despite the potential, don't try to boil the ocean with a large number of IoT use cases. It is important to focus only on a few, selected IoT use cases, in the beginning, to build up momentum and scale. Nevertheless, to become an IoT company, in the long run, you need to transform your entire business and build up IoT-specific capabilities. In the end, designing and manufacturing pure physical products differs significantly from developing software and IoT-enabled products.

6. Start small with a prototype: Building a successful IoT solution comes with many challenges, from simply connecting the devices, managing them 
to monetizing them. Therefore, every IoT endeavour should start with a prototype to validate an idea and to not get lost in potentially unscalable ideas. This is especially true for traditional product companies that are new to agile product development.

7. Develop a long-term roadmap: Oftentimes, companies invest a lot into their digitization efforts (e.g. IoT) but their expectations do not meet their actual returns (Wortmann et al. 2019). This digitalization paradox needs to be kept in mind when dealing with stakeholders. Once kicking off your IoT journey, it is important to develop a set of relevant KPIs (beyond return on investment). In the beginning, the adoption and number of connected devices can serve as good proxies for tracking and managing success. In addition, a long-term strategy for the monetization of the IoT products and services needs to be outlined already at the very beginning.

\section{Lessons Learned for the loT Journey}

- Systematically assess your loT opportunities.

- Define your own role and think about partnerships.

- Stay true to your core.

- Customer value first, technology second.

- Have a holistic loT strategy in mind but prioritize.

- Start small with a prototype.

- Develop a long-term roadmap.

\section{References}

Beecham Research (2020) Why IoT projects fail. Retrieved from https://www.whyiotprojectsfail.com/?cs=br2. Accessed 4 Sept 2020

Bilgeri D, Gebauer H, Fleisch E, Wortmann F (2019) Driving process innovation with IoT field data. MIS Q Exec, 18(3):191-207. https://doi.org/Bosch IoT suite capabilities

Bosch.IO (2020a) Bosch IoT suite. https://www.bosch-iot-suite.com. Accessed 20 Sept 2020

Bosch.IO (2020b) Bosch IoT Suite capabilities. https://www.bosch-iot-suite.com/ capabilities-bosch-iot-suite/. Accessed 20 Sept 2020

Bosch.IO (n.d.) Bosch IoT Suite connects cars, mobile machinery, and baby buggies. https://blog.bosch-si.com/bosch-iot-suite/bosch-iot-suite-connects-cars-mobilemachinery-and-baby-buggies/. Accessed 2 Oct 2020

Boyes H, Hallaq B, Cunningham J, Watson T (2018) The industrial internet of things (IIoT): an analysis framework. Comput Ind 101(June):1-12. https://doi. org/10.1016/j.compind.2018.04.015 
Denner V (2020) Driving change in challenging times. Retrieved from https://boschconnected-world.com/wp-content/uploads/BCW20_Denner_Volkmar.pdf

Fleisch E, Weinberger M, Wortmann F (2014) Business models and the internet of things. Bosch IoT Lab White Paper

Graf J, Krenz W, Kronenwett D (2018) Ilot platforms: Source of profit or inflated hype? How machinery companies should target their investments to maximize benefits. https:/www.oliverwyman.com/content/dam/oliver-wyman/v2/publications/2018/november/perspectives-on-manufacturing-industries-cover-story.pdf. Accessed 19 Sept 2020

Hodapp D, Remane G, Hanelt A, Kolbe L M (2019) Business models for internet of things platforms: empirical development of a taxonomy and archetypes business models for internet of things platforms. In 14th International Conference on Wirtschaftsinformatik

Iansiti M, Lakhani KR (2014) Digital ubiquity how connections, sensors, and data are revolutionizing business. Harv Bus Rev 92(11):90-99

Kranz M (2017) Success with the internet of things requires more than chasing the cool factor. Harv Bus Rev

Mercedes (2020) Mercedes me connect. https://www.me.mercedes-benz.com/passengercars/mercedes-benz-cars/mercedes-me/my-mercedes-me.module.html. Accessed 2 Oct 2020

Porter ME, Heppelmann JE (2014) Smart, connected products are transforming competition. Harv Bus Rev 92(11):64-88

Porter ME, Heppelmann JE (2015) How smart, connected products are transforming companies. Harv Bus Rev 93(10):96-114

Wortmann F, Bilgeri D, Gebauer H, Lamprecht C, Fleisch E (2019) Geld verdienen im IoT - aber wie? HMD Praxis Der Wirtschaftsinformatik 56(6):1094-1112

Sven Jung is a $\mathrm{PhD}$ candidate at the Institute of Technology Management at the University of St. Gallen. He is part of the Bosch IoT Lab, a cooperation between the Bosch Group, University of St. Gallen and ETH Zurich, where he investigates (IoT) platform strategies and platform business models. Sven holds a Master's degree in Finance and Strategic Management from Copenhagen Business School and a Bachelor's degree in Business Administration from the University of St. Gallen. During his studies, he worked for a leading management consultancy, a biotech startup and an investment bank advising technology start-ups.

Stefan Ferber, has been Co-CEO and CTO of Bosch.IO (formerly Bosch Software Innovations $\mathrm{GmbH}$ ) since January 2020. Before that, he was $\mathrm{CEO}$ of Bosch Software Innovations $\mathrm{GmbH}$ and served in several leadership roles at Bosch, including the development of the Bosch IoT Suite and leading a research department. He also represents Bosch on the Board of Directors of the Eclipse Foundation and is a member of the European Internet of Things Council. Stefan Ferber has more than 35 years 
experience in software development, software processes, software product lines, and software architectures for embedded systems, computer vision, and IT domains. Stefan Ferber holds an undergraduate degree and a PhD in computer science from the University of Karlsruhe, Germany and an MSc in computer science from the University of Massachusetts Dartmouth, USA. He is a certified ATAM lead evaluator by the Software Engineering Institute of Carnegie Mellon University, Pittsburgh, USA.

Irene Cramer, Dr., is a Director of Business Development at Software AG. Irene Cramer has worked as a Chief Product Owner and Business Development Manager at Bosch.IO GmbH and is an expert in IoT and industrial applications of Artificial Intelligence and Machine Learning methods. She holds a PhD in Computational Linguistics.

Wolfgang Bronner, Dr., is managing director of the Bosch IoT Lab, a cooperation between the Bosch Group, University of St. Gallen, and ETH Zurich. This lab is particularly dedicated on the Internet of Things, Data Science, and Business Model Innovation. Wolfgang Bronner had his first insight into the high-tech industry as a student at IBM in Silicon Valley. He has been working as an expert and manager in various industries and functions at Bosch in Germany and the USA since 2002. In his last position, he analyzed the impact of the most significant future technologies such as AI on Bosch, derived strategic recommendations, and discussed them with the executive management. He received his $\mathrm{PhD}$ in physics from the University of Stuttgart in 2003.

Felix Wortmann, Prof. Dr., is Assistant Professor of Technology Management at the University of St. Gallen. He is also the Scientific Director of the Bosch IoT Lab, a cooperation between the Bosch Group, University of St. Gallen, and ETH Zurich. Felix Wortmann has published more than 100 publications and is among the top 5\% of all scientists in business administration in the German-speaking countries (Wirtschaftswoche 2019). His research interests include the Internet of Things, machine learning, blockchain, and business model innovation in manufacturing, mobility, healthcare, and energy. From 2006 to 2009 he worked as an assistant to the executive board of SAP. After studying information systems, Felix Wortmann received his PhD in Management from the University of St. Gallen in 2006. 